\title{
Recollision as a probe of magnetic-field effects in nonsequential double ionization
}

\author{
A. Emmanouilidou and T. Meltzer \\ Department of Physics and Astronomy, University College London, Gower Street, London WC1E 6BT, United Kingdom
}

(Received 2 December 2016; published 7 March 2017)

\begin{abstract}
Fully accounting for nondipole effects in the electron dynamics, double ionization is studied for He driven by a near-infrared laser field and for Xe driven by a mid-infrared laser field. Using a three-dimensional semiclassical model, the average sum of the electron momenta along the propagation direction of the laser field is computed. If nondipole effects are not accounted for, the average momentum of each electron in the propagation direction of the laser field is zero. When nondipole effects are accounted for the sum of the electron momenta is found to be an order of magnitude larger than twice the average electron momentum along the propagation direction of the laser field in single ionization. This average sum of the electron momenta is found to be maximum at intensities smaller than the intensities satisfying previously predicted criteria for the onset of magnetic-field effects. It is shown that strong recollisions are the reason for this unexpectedly large value of the sum of the momenta along the direction of the magnetic component of the Lorentz force.
\end{abstract}

DOI: 10.1103/PhysRevA.95.033405

\section{INTRODUCTION}

Nonsequential double ionization (NSDI) in two-electron atoms is a fundamental process that explores electron-electron correlation in strong fields. As such, it has attracted a lot of interest in the field of light-matter interactions in recent years $[1,2]$. The majority of theoretical studies on NSDI are delivered in the framework of the dipole approximation, particularly the studies involving the commonly used near-infrared laser fields and intensities [3]. In the dipole approximation the vector potential $\mathbf{A}$ of the laser field does not depend on space. Therefore, magnetic-field effects are neglected since the magnetic-field component of the laser field $\mathbf{B}=\boldsymbol{\nabla} \times \mathbf{A}(t)$ is zero. However, in the general case where $\mathbf{A}$ depends both on space and time, an electron experiences a Lorentz force whose magnetic-field component $\mathbf{F}_{\mathbf{B}}$ increases with increasing electron velocity since $\mathbf{F}_{\mathbf{B}}=q \mathbf{v} \times \mathbf{B}$. It is important to account for magnetic-field effects since in strong-field ionization high-velocity electrons are often produced. Criteria for the onset of magnetic-field effects in both the relativistic and nonrelativistic limits have already been formulated $[4,5]$. In the nonrelativistic limit, where this work focuses, magnetic-field effects are expected to arise when the amplitude of the electron motion due to the magnetic-field component of the Lorentz force becomes 1 a.u., i.e. $\beta_{0} \approx U_{p} /(2 \omega c) \approx 1$ a.u. [4,5], with $U_{p}$ being the ponderomotive energy.

Studies addressing magnetic-field effects include using a three-dimensional (3D) semiclassical rescattering model that accounts for $\mathbf{F}_{\mathbf{B}}$ to successfully describe the observed ionization of $\mathrm{Ne}^{n+}(n \leqslant 8)$ in ultrastrong fields [6]. Moreover, nondipole effects were addressed in theoretical studies of stabilization [7] and high-order-harmonic generation by neglecting the Coulomb potential [8] by using a first-order expansion of the vector potential [9] or by using a Monte Carlo simulation [10]. In recent studies of single ionization (SI), the electron momentum distribution along the propagation direction of the laser field was computed using different quantum-mechanical approaches [11-14]. For example, for $\mathrm{H}$ interacting with a $3400-\mathrm{nm}$ laser field at intensities $(0.5-1) \times 10^{14} \mathrm{~W} \mathrm{~cm}^{-2}$ the average momentum along the propagation direction of the laser field was found to increase from 0.003 to 0.006 a.u. [12]. Thus, for single ionization, the average of this momentum component increases with increasing $\beta_{0}[12,15]$. If magnetic-field effects are not accounted for, then this momentum component averages to zero. The motivation for these theoretical studies was a recent experimental observation of the average momentum in the propagation direction of the laser field [15].

This work reveals another aspect of NSDI which has not been previously addressed. The strong electron-electron correlation in NSDI is identified as a probe of magneticfield effects for both near-infrared and mid-infrared intense laser fields. Specifically, the intensities considered are around $10^{15} \mathrm{~W} \mathrm{~cm}^{-2}$ for $\mathrm{He}$ at $800 \mathrm{~nm}$ and around $10^{14} \mathrm{~W} \mathrm{~cm}^{-2}$ for $\mathrm{Xe}$ at $3100 \mathrm{~nm}$, where the rescattering mechanism underlies double ionization [16]. For these intensities, it is found that the average sum of the two electron momenta along the propagation direction of the laser field is unexpectedly large. It is roughly an order of magnitude larger than twice the average of the respective electron momentum for single ionization. This average sum of the momenta for double ionization (DI) is shown to be maximum at intensities smaller than the intensities satisfying the criterion for the onset of magnetic-field effects $\beta_{0} \approx 1$ a.u. [4,5]. This is illustrated in Fig. 1 for He driven by a near-infrared $(800-\mathrm{nm})$ laser field and for Xe driven by a mid-infrared $(3100-\mathrm{nm})$ laser field. The motivation for choosing near-infrared laser fields is that they are very common in strong field studies. Mid-infrared laser fields are chosen because magnetic-field effects set in for small intensities (see Fig. 1), attracting a lot of interest in recent years [17,18].

\section{METHOD}

For the current studies, a 3D semiclassical model is employed that fully accounts for nondipole effects during the time propagation. For simplicity this model is referred to as 3D-SMND. It is an extension of a 3D semiclassical model that was previously formulated in the framework of the dipole approximation. This latter model is referred to as 3D-SMD. Thus, in the 3D-SMND model nondipole effects are fully accounted for in the two-electron dynamics. Some of the successes of the 3D-SMD model are identifying 


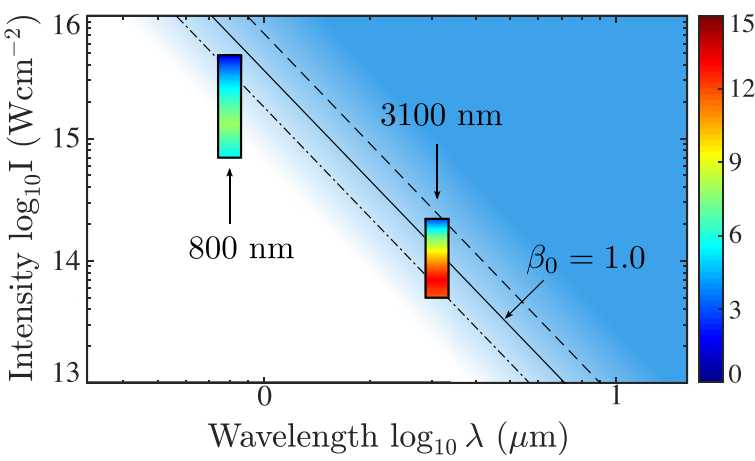

FIG. 1. Range of validity of the dipole approximation and momentum in double ionization. The white area indicates the range of intensities and wavelengths where the dipole approximation is valid. $\beta_{0}=0.5$ a.u. (dot-dashed line), $\beta_{0}=1$ a.u. (solid line), and $\beta_{0}=2$ a.u. (dashed line). The arrows mark the 800- and 3100-nm wavelengths driving $\mathrm{He}$ and $\mathrm{Xe}$, respectively. At these wavelengths, for a range of intensities, the color bars indicate the ratio of the average sum of the electron momenta along the direction of $\mathbf{F}_{\mathbf{B}}$ for double ionization with twice the respective electron momentum for single ionization $\left\langle p_{y}^{1}+p_{y}^{2}\right\rangle_{\mathrm{DI}} /\left(2\left\langle p_{y}\right\rangle_{\mathrm{SI}}\right)$.

the mechanism responsible for the fingerlike structure [19], which was predicted theoretically [20] and was observed experimentally for He driven by 800 -nm laser fields [21,22]; investigating direct versus delayed pathways of NSDI for $\mathrm{He}$ driven by a 400-nm laser field while achieving excellent agreement with fully $a b$ initio quantum-mechanical calculations [23]; identifying the underlying mechanisms for the carrierenvelope phase effects observed experimentally in NSDI of Ar driven by an $800-\mathrm{nm}$ laser field at a range of intensities [24,25]. The 3D-SMD model is extended to the 3D-SMND model employed in the current work to fully account for the magnetic field during time propagation. The Hamiltonian describing the interaction of the fixed-nucleus two-electron atom with the laser field is given by

$$
\begin{aligned}
H= & \frac{\left[\mathbf{p}_{1}+\mathbf{A}\left(y_{1}, t\right)\right]^{2}}{2}+\frac{\left[\mathbf{p}_{2}+\mathbf{A}\left(y_{2}, t\right)\right]^{2}}{2} \\
& -c_{1} \frac{Z}{\left|\mathbf{r}_{1}\right|}-c_{2} \frac{Z}{\left|\mathbf{r}_{2}\right|}+c_{3} \frac{1}{\left|\mathbf{r}_{1}-\mathbf{r}_{2}\right|},
\end{aligned}
$$

where the vector potential $\mathbf{A}$ is given by

$$
\mathbf{A}(y, t)=-\frac{E_{0}}{\omega} e^{-\left(\frac{c t-y}{c \tau}\right)^{2}} \sin (\omega t-k y) \hat{x},
$$

$\omega, k, E_{0}$ are the frequency, wave number, and strength of the electric component of the laser field, respectively, and $c$ is the velocity of light. $\tau=$ FWHM $/ \sqrt{\ln 4}$ with FWHM being the full width half maximum of the laser field. All Coulomb forces are accounted for by setting $c_{1}=c_{2}=c_{3}=1$. In this work linearly polarized laser fields are considered. To switch off a Coulomb interaction we set the appropriate constant equal to zero; for example, to switch off the interaction of electron 1 with the nucleus we set $c_{1}=0$. For $\mathbf{A}$ given by Eq. (2), $\mathbf{E}$ and $\mathbf{B}$ are along the $x$ and $z$ axes, respectively, while the propagation direction of the laser field and the direction of $\mathbf{F}_{\mathbf{B}}$ are along the $y$ axis. Unless otherwise stated, all Coulomb forces as well as the electric and the magnetic fields are fully accounted for during time propagation. Moreover, the Coulomb singularity is addressed using regularized coordinates [26] which were also employed in the 3D-SMD model $[19,23,24]$.

The initial state in the 3D-SMND model is taken to be the same as in the 3D-SMD model $[19,23,24]$. It entails one electron tunneling through the field-lowered Coulomb potential with a nonrelativistic quantum tunneling rate given by the Ammosov-Delone-Krainov (ADK) formula [27,28]. The momentum along the direction of the electric field is zero, while the transverse one is given by a Gaussian distribution [27,28]. A nonrelativistic ADK rate results in this Gaussian distribution being centered around zero. In Ref. [29] nondipole effects were accounted for in the ADK rate. It was shown that the most probable transverse velocity ranges from $0.33 I_{p} / c$ to almost zero with increasing $E_{0} /\left(2 I_{p}\right)^{3 / 2}$, with $I_{p}$ being the ionization energy of the tunneling electron. In this work, the smallest intensities considered are $5 \times 10^{13} \mathrm{~W} \mathrm{~cm}^{-2}$ for $\mathrm{Xe}$ and $7 \times 10^{14} \mathrm{~W} \mathrm{~cm}^{-2}$ for He. At these intensities, if nondipole effects are accounted for in the ADK rate, the transverse velocity of the tunneling electron is centered around $0.17 I_{p}^{\mathrm{Xe}} / c$ for $\mathrm{Xe}$, which is $5.5 \times 10^{-4}$ a.u. $\left(I_{p}^{\mathrm{Xe}}=0.446\right.$ a.u. $)$, and $0.12 I_{p}^{\mathrm{He}} / c$ for He, which is $7.9 \times 10^{-4}$ a.u. $\left(I_{p}^{\mathrm{He}}=0.904\right.$ a.u. $)$. These values are significantly smaller than the values of the average momenta along the propagation direction of the laser field, which are presented in what follows. Thus, using the nonrelativistic ADK rate is a good approximation for the quantities addressed in this work. The remaining electron is initially described by a microcanonical distribution [30]. In what follows, the tunneling and bound electrons are denoted as electrons 1 and 2, respectively.

\section{RESULTS}

\section{A. $p_{y}$ for single ionization of $\mathrm{Xe}$ and $\mathrm{H}$}

The accuracy of the 3D-SMND model is established by computing the momentum distribution along the propagation direction of the laser field $p_{y}$ for SI and by comparing it with available experimental and theoretical results. In Ref. [18], the peak of the $p_{y}$ distribution was observed to shift in the direction opposite to the magnetic-field component of the Lorentz force $\mathbf{F}_{\mathbf{B}}$ for intensities of the order of $10^{13} \mathrm{~W} \mathrm{~cm}^{-2}$. This shift was attributed to the combined effect of the magnetic field and the Coulomb attraction of the nucleus [18]. To compare with these experimental results, the shift of the peak of the $p_{y}$ distribution is computed for Xe interacting with a $3400-\mathrm{nm}$ and $44-\mathrm{fs}$ FWHM laser field as the intensity increases from $3 \times 10^{13}$ to $6 \times 10^{13} \mathrm{~W} \mathrm{~cm}^{-2}$. The shift of the peak of the $p_{y}$ distribution is found to vary from -0.0055 to -0.012 a.u. These results are in agreement with the simulations and experimental results presented in Ref. [18]. Moreover, to compare with the results in Ref. [12], the average of the momentum $\left\langle p_{y}\right\rangle_{\mathrm{SI}}$ is computed for $\mathrm{H}$ driven by a 3400-nm and 16-fs FWHM laser field for intensities $(0.5-1) \times 10^{14} \mathrm{~W} \mathrm{~cm}^{-2}$. Using the 3D-SMND model, $\left\langle p_{y}\right\rangle_{\mathrm{SI}}$ is found to vary from 0.0022 to 0.0046 a.u. These values differ by $27 \%$ from the results presented in Ref. [12] and are thus in reasonable agreement. The difference may be due to nondipole effects not accounted for in the ADK rate in the 3D-SMND model. In addition, the quantum calculation used in Ref. [12] employs a two-dimensional soft-core potential, while 
TABLE I. Single-ionization results for Xe and He.

\begin{tabular}{|c|c|c|c|c|c|c|c|}
\hline & & \multicolumn{2}{|c|}{$\begin{array}{l}\text { SI } Z=2, \\
c_{1,2,3}=1\end{array}$} & \multicolumn{2}{|c|}{$\begin{array}{c}\mathrm{SI} \\
c_{1,2,3}=0\end{array}$} & \multicolumn{2}{|c|}{$\begin{array}{c}\text { SI } Z=2, \\
c_{1}=0, c_{2,3}=1\end{array}$} \\
\hline \multicolumn{2}{|c|}{$I\left(10^{15} \mathrm{~W} \mathrm{~cm}^{-2}\right)$} & $\left\langle p_{y}\right\rangle^{\mathrm{a}}$ & $\left\langle E_{k} / c\right\rangle^{\mathrm{a}}$ & $\left\langle p_{y}\right\rangle^{\mathrm{a}}$ & $\left\langle E_{k} / c\right\rangle^{\mathrm{a}}$ & $\left\langle p_{y}\right\rangle^{\mathrm{a}}$ & $\left\langle E_{k} / c\right\rangle^{\mathrm{a}}$ \\
\hline \multirow[t]{3}{*}{$\mathrm{He}$} & 0.7 & 3.5 & 2.3 & 1.5 & 1.5 & 1.8 & 2.2 \\
\hline & 1.3 & 6.0 & 4.4 & 3.4 & 3.4 & 3.4 & 4.4 \\
\hline & 4.8 & 28 & 32 & 19 & 19 & 19 & 21 \\
\hline \multirow[t]{3}{*}{$\mathrm{Xe}$} & 0.05 & 3.2 & 1.6 & 1.3 & 1.3 & 1.8 & 1.6 \\
\hline & 0.07 & 3.5 & 2.5 & 2.1 & 2.1 & 2.4 & 2.4 \\
\hline & 0.22 & 11 & 12 & 9.9 & 9.9 & 9.1 & 11 \\
\hline
\end{tabular}

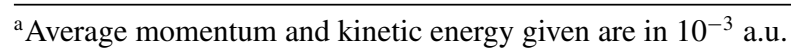

a full 3D potential is employed by the 3D-SMND model. The single-ionization results obtained in this work were computed with at least $4 \times 10^{5}$ events, and therefore, the statistical error introduced is very small.

\section{B. $\left\langle p_{y}\right\rangle$ for single ionization of $\mathrm{He}$ and $\mathrm{Xe}$}

The 3D-SMND model is now employed to compute $\left\langle p_{y}\right\rangle_{\mathrm{SI}}$ for He driven by an 800-nm, 12-fs FWHM laser field and for Xe driven by a 3100-nm, 44-fs FWHM laser field; the two laser fields have roughly the same number of cycles. First, an analytic expression is obtained relating $\left\langle p_{y}\right\rangle_{\mathrm{SI}}$ to the average electron kinetic energy $\left\langle E_{k}\right\rangle_{\mathrm{SI}}[11,15]$. When an electron interacts with an electromagnetic field with all the Coulomb forces switched off, i.e., $c_{1}=c_{2}=c_{3}=0$ in Eq. (1), the equations of motion are $\dot{p}_{y}=-(\mathbf{v} \times \mathbf{B})_{y}$ and $\dot{p}_{x}=-(\mathbf{v} \times \mathbf{B})_{x}-E$. Keeping only first-order terms in $1 / c$, $\dot{p}_{x}=-E$, resulting in $p_{y}-p_{0, y}=p_{x}^{2} /(2 c)-p_{0, x}^{2} /(2 c)$, with $p_{0, x / y}$ being the $x$ and $y$ components of the electron momentum at time $\mathrm{t}_{0}$. The initial momentum of the tunneling electron along the electric-field direction is set to zero, as in the 3D-SMND model, resulting in $p_{y}-p_{0, y}=p_{x}^{2} /(2 c)=E_{k} / c$. Thus, $\left\langle p_{y}\right\rangle=\left\langle p_{0, y}\right\rangle+\left\langle E_{k}\right\rangle / c$ is obtained. For this simple model $\left\langle E_{k}\right\rangle$ is the drift energy of the electron. As discussed in Sec. II, if nondipole effects are accounted for in the tunneling rate, then $\left\langle p_{0, y}\right\rangle$ varies from $0.33 I_{p} / c$ to almost zero with increasing intensity. In the 3D-SMND model nondipole effects are not included in the ADK rate, and therefore, $\left\langle p_{0, y}\right\rangle=0$. Indeed, using the 3D-SMND model with $c_{1}=c_{2}=c_{3}=0$, it is found that $\left\langle p_{y}\right\rangle_{S I}=\left\langle E_{k}\right\rangle_{S I} / c$ (see Table I).

Next, $\left\langle p_{y}\right\rangle_{\mathrm{SI}}$ and $\left\langle E_{k}\right\rangle_{\mathrm{SI}}$ are computed with the 3D-SMND model fully accounting for all Coulomb forces and the presence of the initially bound electron in driven $\mathrm{He}$ and $\mathrm{Xe}$, i.e., $c_{1}=c_{2}=c_{3}=1$ with $Z=2$. The tunneling electron is the one that is mostly singly ionizing. In Fig. 2, we show that, for $\mathrm{He},\left\langle p_{y}\right\rangle_{\mathrm{SI}}$ varies from 0.0035 to 0.028 a.u. at intensities $(0.7-4.8) \times 10^{15} \mathrm{~W} \mathrm{~cm}^{-2}$. For $\mathrm{Xe},\left\langle p_{y}\right\rangle_{\mathrm{SI}}$ varies from 0.0032 to 0.011 a.u. at intensities $(0.5-2.2) \times 10^{14} \mathrm{~W} \mathrm{~cm}^{-2}$.

In Table I, it is shown that $\left\langle p_{y}\right\rangle_{\mathrm{SI}}$ and $\left\langle E_{k}\right\rangle_{\mathrm{SI}} / c$ when obtained with the full model do not differ by more than a factor of 3 from the values obtained when all Coulomb forces are switched off. Thus, the simple model yields the correct order of magnitude for $\left\langle p_{y}\right\rangle_{\mathrm{SI}}$. It is also shown in Table I that with all Coulomb forces accounted for, $\left\langle p_{y}\right\rangle_{\mathrm{SI}}$ is no longer equal to $\left\langle E_{k}\right\rangle_{\mathrm{SI}} / c$ for either driven He or driven Xe. For the full model, $\left\langle E_{k}\right\rangle_{\mathrm{SI}}$ is no longer just the drift kinetic energy, mainly due to the interaction of the tunneling electron with the nucleus. Indeed, using the 3D-SMND model with this interaction switched off, i.e., $c_{1}=0$ and $c_{2}=c_{3}=1,\left\langle p_{y}\right\rangle_{\mathrm{SI}}$ is roughly equal to $\left\langle E_{k}\right\rangle_{\mathrm{SI}} / c$ (see Table I). $\left\langle p_{y}\right\rangle_{\mathrm{SI}}$ is also shown in Table I to be more sensitive than $\left\langle E_{k}\right\rangle_{\mathrm{SI}}$ to the interaction of the tunneling electron with the nucleus. Summarizing the results for single ionization, propagating classical trajectories with initial times determined by the ADK rate and all Coulomb forces switched off yields the correct order of magnitude for $\left\langle p_{y}\right\rangle_{\mathrm{SI}}$.

\section{C. $\left\langle p_{y}^{1}+p_{y}^{2}\right\rangle$ for double ionization of He and Xe}

For double ionization, the average of the sum of the electron momenta along the propagation direction of the laser field $\left\langle p_{y}^{1}+p_{y}^{2}\right\rangle_{\text {DI }}$ is computed for He driven by an 800-nm laser field and for Xe driven by a 3100-nm laser field. The parameters of the laser fields are the same as the ones employed in the single-ionization section for $\mathrm{He}$ and $\mathrm{Xe}$. The double-ionization results obtained in this work were computed with at least $2 \times 10^{5}$ events, and therefore, the statistical error introduced is very small. The results are plotted in Fig. 3(a) for He at intensities of $(0.7-4.8) \times 10^{15} \mathrm{~W} \mathrm{~cm}^{-2}$ and in Fig. 3(b) for $\mathrm{Xe}$ at intensities of $(0.5-2.2) \times 10^{14} \mathrm{~W} \mathrm{~cm}^{-2}$. The values obtained for $\left\langle p_{y}^{1}+p_{y}^{2}\right\rangle_{\text {DI }}$ are quite unexpected. Specifically,
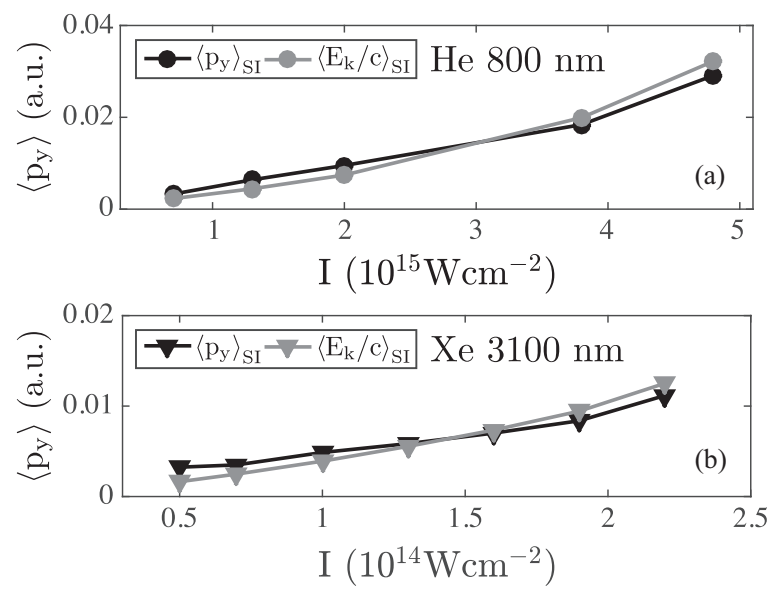

FIG. 2. Single ionization of He and Xe. $\left\langle p_{y}\right\rangle_{\mathrm{SI}}$ and $\left\langle E_{k}\right\rangle_{\mathrm{SI}} / c$ are plotted as a function of intensity in (a) for He driven by an $800-\mathrm{nm}$ laser field and in (b) for Xe driven by a 3100-nm laser field. 

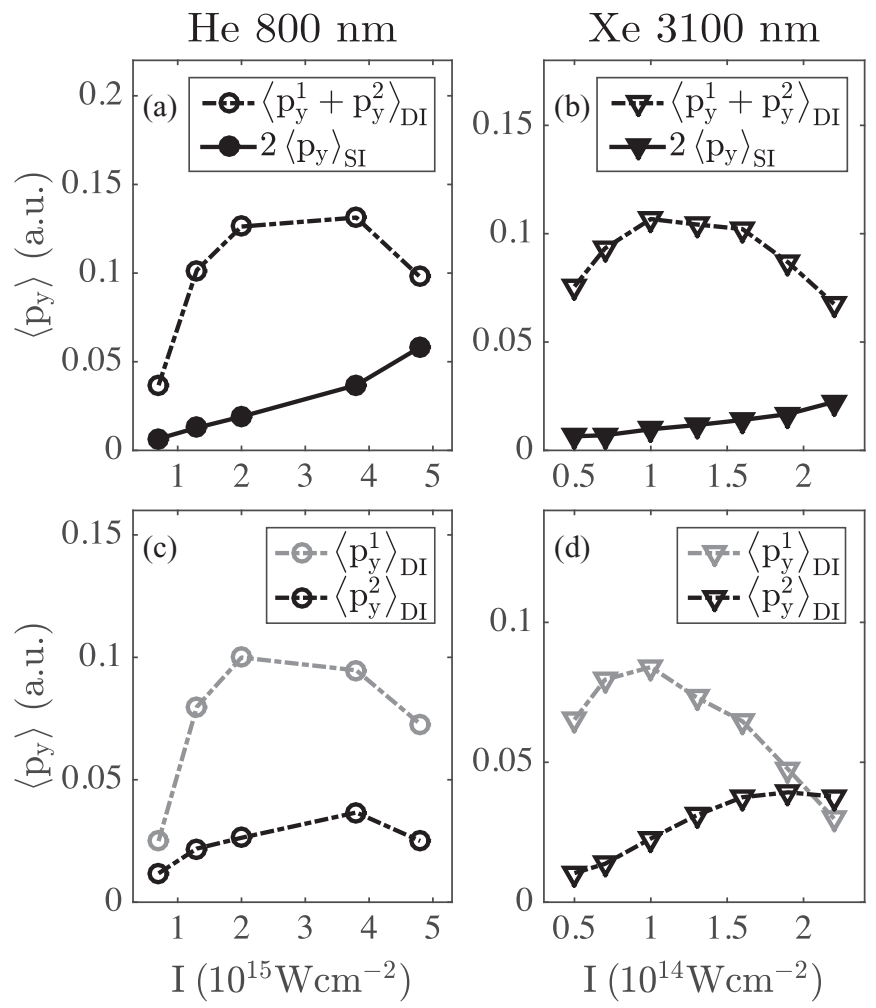

FIG. 3. Double ionization of He and Xe. $\left\langle p_{y}^{1}+p_{y}^{2}\right\rangle_{\mathrm{DI}}$ and $2\left\langle p_{y}\right\rangle_{\mathrm{SI}}$ are plotted as a function of intensity in (a) for He driven by an 800-nm laser field and in (b) for Xe driven by a 3100-nm laser field. $\left\langle p_{y}^{1}\right\rangle_{\mathrm{DI}}$ and $\left\langle p_{y}^{2}\right\rangle_{\mathrm{DI}}$ are plotted as a function of intensity in (c) for He driven by an $800-n m$ laser field and in (d) for Xe driven by a 3100-nm laser field.

$\left\langle p_{y}^{1}+p_{y}^{2}\right\rangle_{\mathrm{DI}}$ is found to be roughly an order of magnitude larger than twice $\left\langle p_{y}\right\rangle_{\mathrm{SI}}$, with $\left\langle p_{y}\right\rangle_{\mathrm{SI}}$ computed in the previous section. For comparison, both $\left\langle p_{y}^{1}+p_{y}^{2}\right\rangle_{\mathrm{DI}}$ and $2\left\langle p_{y}\right\rangle_{\mathrm{SI}}$ are displayed in Fig. 3. It is shown that $\left\langle p_{y}^{1}+p_{y}^{2}\right\rangle_{\mathrm{DI}} \approx 8 \times 2\left\langle p_{y}\right\rangle_{\mathrm{SI}}$ for He at $1.3 \times 10^{15} \mathrm{~W} \mathrm{~cm}^{-2}$, while $\left\langle p_{y}^{1}+p_{y}^{2}\right\rangle_{\mathrm{DI}} \approx 13 \times 2\left\langle p_{y}\right\rangle_{\mathrm{SI}}$ for $\mathrm{Xe}$ at $7 \times 10^{13} \mathrm{~W} \mathrm{~cm}^{-2}$. For $1.3 \times 10^{15} \mathrm{~W} \mathrm{~cm}^{-2}$ and 800 $\mathrm{nm} \beta_{0}=0.18$ a.u., while for $7 \times 10^{13} \mathrm{~W} \mathrm{~cm}^{-2}$ and 3100 $\mathrm{nm} \beta_{0}=0.58$ a.u. Thus, $\left\langle p_{y}^{1}+p_{y}^{2}\right\rangle_{D I} / 2\left\langle p_{y}\right\rangle_{S I}$ is found to be maximum at intensities considerably smaller than the intensities corresponding to $\beta_{0} \approx 1$ a.u., i.e., the criterion for the onset of magnetic-field effects [4,5]. This is shown in Fig. 1. Moreover, unlike $\left\langle p_{y}\right\rangle_{\text {SI }}$, which increases with increasing intensity as expected [12], $\left\langle p_{y}^{1}+p_{y}^{2}\right\rangle_{D I}$ after reaching a maximum decreases with increasing intensity for the range of intensities currently considered [Figs. 3(a) and 3(b)]. What is the mechanism responsible for this pattern? We answer this question in what follows.

\section{Recollision probing magnetic-field effects}

The average electron momentum along the propagation direction is nonzero when the magnetic-field component of the Lorentz force $\mathbf{F}_{\mathbf{B}}$ is accounted for. This force increases with increasing intensity (increasing strength of the magnetic field) and with increasing velocity along the direction of the electric field. $\left\langle p_{y}^{1}+p_{y}^{2}\right\rangle_{\mathrm{DI}} / 2\left\langle p_{y}\right\rangle_{\mathrm{SI}}$ is found to be maximum at $1.3 \times 10^{15} \mathrm{~W} \mathrm{~cm}^{-2}$ for $800 \mathrm{~nm}$ and at $7 \times 10^{13} \mathrm{~W} \mathrm{~cm}^{-2}$ for $3100 \mathrm{~nm}$, intensities where the strength of the magnetic field is not large. It then follows that it must be the velocities of the two escaping electrons that are large at these intensities. Large electron velocities at intermediate intensities are a result of strong electron-electron correlation, i.e., of the rescattering mechanism [16]. In the rescattering scenario after electron 1 tunnels in the field-lowered Coulomb potential, it accelerates in the strong laser field and can return to the core and undergo a collision with the remaining electron [16]. In what follows evidence is provided that the large values of $\left\langle p_{y}^{1}+p_{y}^{2}\right\rangle_{\mathrm{DI}} / 2\left\langle p_{y}\right\rangle_{\mathrm{SI}}$ are due to recollisions. Specifically, it is shown that recollisions are strong, resulting in overall large kinetic energies compared to the ponderomotive energy $U_{p}$, which is given by $E_{0}^{2} /\left(4 \omega^{2}\right)$, roughly at the intensities where $\left\langle p_{y}^{1}+p_{y}^{2}\right\rangle_{\mathrm{DI}} / 2\left\langle p_{y}\right\rangle_{\mathrm{SI}}$ is maximum. It is also shown that recollisions are soft, resulting in overall smaller kinetic energies compared to the ponderomotive energy $U_{p}$ at higher intensities where $\left\langle p_{y}^{1}+p_{y}^{2}\right\rangle_{\mathrm{DI}} / 2\left\langle p_{y}\right\rangle_{\mathrm{SI}}$ is found to be smaller.

This transition from strong to soft recollisions is demonstrated in the context of He driven by an $800-\mathrm{nm}$ laser field at intensities $0.7 \times 10^{15}, 2.0 \times 10^{15}$, and $3.8 \times 10^{15} \mathrm{~W} \mathrm{~cm}^{-2}$. To do so, an analysis of the double-ionization events is performed. It is noted that a similar analysis was found to hold for Xe (not shown) where strong recollisions prevail at intensities $0.7 \times 10^{14}$ and $10^{14} \mathrm{~W} \mathrm{~cm}^{-2}$, while soft ones prevail at $2.2 \times$ $10^{14} \mathrm{~W} \mathrm{~cm}^{-2}$. Focusing on He, in Fig. 4, the distribution of the tunneling and recollision times is plotted. For the intensities $0.7 \times 10^{15}, 2.0 \times 10^{15}$, and $3.8 \times 10^{15} \mathrm{~W} \mathrm{~cm}^{-2}$ considered in Fig. 4 the ponderomotive energy $U_{p}$ is equal to $1.54,4.39$, and 8.35 a.u., respectively. Thus, the maximum recollision energies $3.17 U_{p}[16]$ are well above the second ionization energy of $\mathrm{He}$, which is 2 a.u. As expected, for the smaller intensities [Figs. 4(a1) and 4(b1)], electron 1 tunnel ionizes at times around the extrema of the laser field. For $3.8 \times 10^{15} \mathrm{~W} \mathrm{~cm}^{-2}$ [Fig. 4(c1)] the electric field is sufficiently strong that electron 1 can tunnel ionize at times other than the extrema of the field. The distribution of the recollision times is also plotted. This time is identified for each double-ionization trajectory as the time that the electron-electron potential energy $1 /\left|\mathbf{r}_{1}-\mathbf{r}_{2}\right|$ as a function of time is maximum. For the smaller intensities the recollision times are centered roughly around $\pm 2 n T / 3$, with $n$ being an integer and $T$ being the period of the laser field, as expected from the rescattering model [16] [Figs. 4(a2) and 4 (b2)]. At $3.8 \times 10^{15} \mathrm{~W} \mathrm{~cm}^{-2}$ the recollision times shift and are centered around the extrema of the laser field [Fig. 4(c2)]. This shift of the recollision times signals a transition from strong to soft recollisions [31]. This transition is further corroborated by the average kinetic energy of each electron $\left\langle E_{k}^{1,2}\right\rangle$, plotted in Fig. 4 as a function of time; zero time is set equal to the recollision time of each double-ionization trajectory. For smaller intensities, $\left\langle E_{k}^{1,2}\right\rangle$ changes sharply at the recollision time [Figs. 4(a3) and 4(b3)]. The change in $\left\langle E_{k}^{1,2}\right\rangle$ is much smaller at $3.8 \times 10^{15} \mathrm{~W} \mathrm{~cm}^{-2}$ [Fig. 4(c3)]. The above results show that for the smaller intensities electron 1 tunnel ionizes around the extrema of the field. It then returns to the core, roughly when the electric field is small, with large velocity and undergoes a recollision with electron 2 , transferring a large amount of energy (strong recollision). 

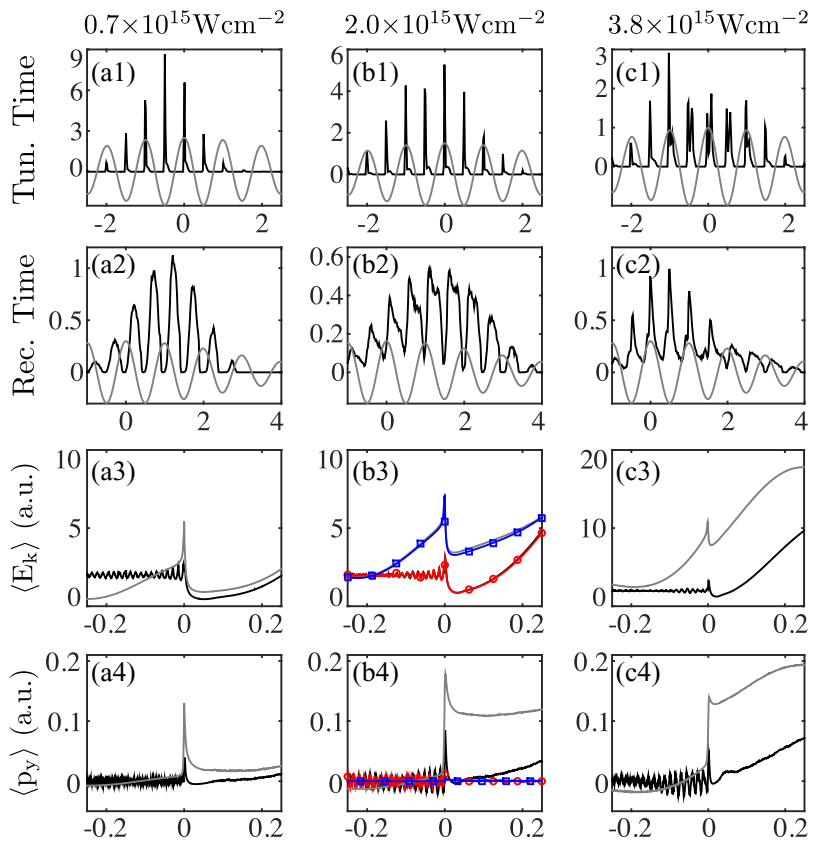

Time (Cycles)
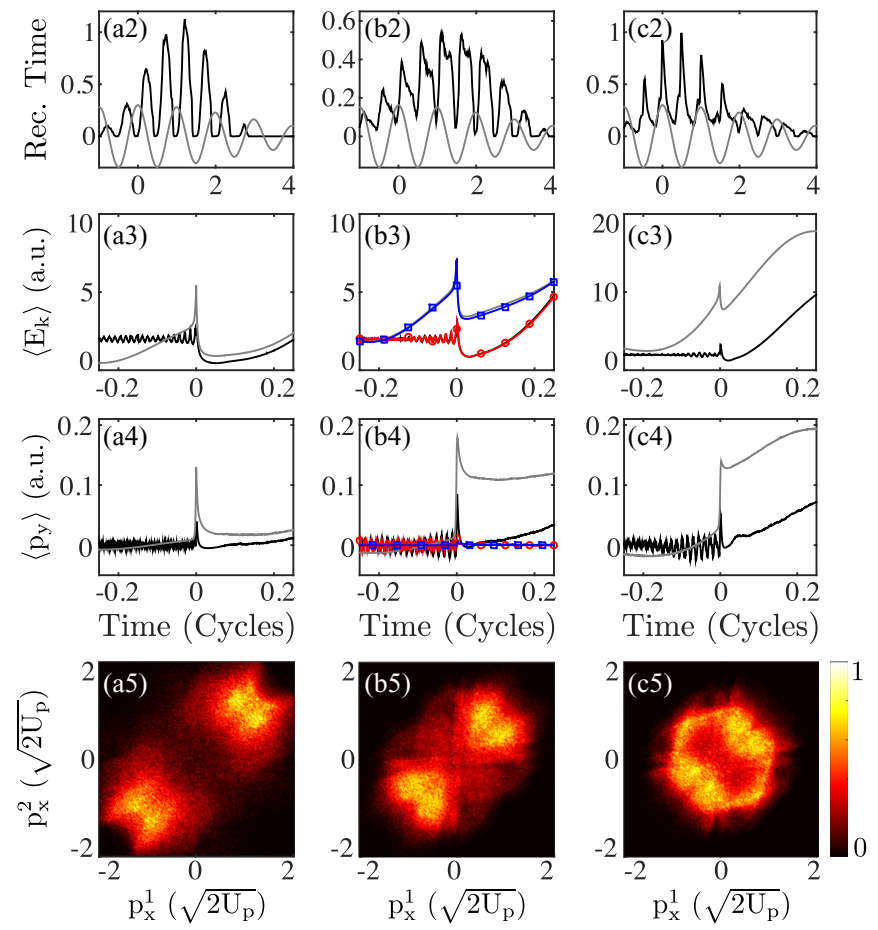

FIG. 4. Recollision underlying double ionization of $\mathrm{He}$ driven at $800 \mathrm{~nm}$. The intensities considered are (a) $0.7 \times 10^{15} \mathrm{~W} \mathrm{~cm}^{-2}$, (b) $2.0 \times 10^{15} \mathrm{~W} \mathrm{~cm}^{-2}$, and (c) $3.8 \times 10^{15} \mathrm{~W} \mathrm{~cm}^{-2}$. The distribution of tunneling times (black line) is plotted in (a1), (b1), and (c1). The distribution of recollision times (black line) is plotted in (a2), (b2), and (c2). The electric field is denoted as a gray line in the plots of the tunneling and recollision times. $\left\langle E_{k}^{1}\right\rangle$ (gray line) and $\left\langle E_{k}^{2}\right\rangle$ (black line) are plotted as a function of time in (a3), (b3), and (c3) with time zero set equal to the recollision time of each double-ionization event. For $2.0 \times 10^{15} \mathrm{~W} \mathrm{~cm}^{-2},\left\langle E_{k}^{1}\right\rangle$ (blue line) and $\left\langle E_{k}^{2}\right\rangle$ (red line) are also plotted in the absence of the magnetic field. $\left\langle p_{y}^{1}\right\rangle$ (gray line) and $\left\langle p_{y}^{2}\right\rangle$ (black line) are plotted as a function of time in (a4), (b4), and (c4) with time zero set equal to the recollision time of each double-ionization event. For $2.0 \times 10^{15} \mathrm{~W} \mathrm{~cm}^{-2},\left\langle p_{y}^{1}\right\rangle$ (blue line) and $\left\langle p_{2}^{2}\right\rangle$ (red line) are also plotted in the absence of the magnetic field. Correlated momenta along the direction of the electric field are plotted in (a5), (b5), and (c5).

The strong recollisions result in higher asymptotic in time kinetic energies of the electrons compared to $U_{p}$. The ratios $\left\langle E_{k, x}^{1}\right\rangle / U_{p}$ and $\left\langle E_{k, x}^{2}\right\rangle / U_{p}$, where $\left\langle E_{k, x}^{1,2}\right\rangle$ are the asymptotic in time average kinetic energies of electrons 1 and 2 along the polarization direction of the laser field, are $117 \%$ and $104 \%$ for an intensity of $0.7 \times 10^{15} \mathrm{~W} \mathrm{~cm}^{-2}$ and $50 \%$ and $77 \%$ for an intensity of $2.0 \times 10^{15} \mathrm{~W} \mathrm{~cm}^{-2}$. The velocities of both electrons along the direction of the electric field are determined mainly by the vector potential at the recollision time. Thus, both electrons escape mainly either parallel or antiparallel to the electric field. Indeed, this is the pattern seen in the plots of the correlated momenta along the direction of the electric field in Figs. 4(a5) and 4(b5), where the highest density is in the first and third quadrants. The correlated momenta are plotted in units of $\sqrt{2 U_{p}}$. These patterns of the correlated momenta are consistent with direct double ionization, that is, with both electrons ionizing shortly after recollision takes place [32]. Indeed, analyzing the double-ionization events, it is found that for $\mathrm{He}$ at $0.7 \times 10^{15} \mathrm{~W} \mathrm{~cm}^{-2}$ direct double ionization contributes $70 \%$. Delayed double-ionization events contribute $30 \%$. In delayed double ionization, also known as recollision-induced excitation with subsequent field ionization $[32,33]$, one electron ionizes soon after recollision takes place, while the other electron ionizes with a delay [23]. In contrast, at the higher intensity of $3.8 \times 10^{15} \mathrm{~W} \mathrm{~cm}^{-2}$, electron 1 tunnel ionizes after the extrema of the laser field. It then follows a short trajectory and returns to the core when the electric field is maximum with small velocity. Electron 1 transfers a small amount of energy to electron 2 (soft recollision). Indeed, the ratios $\left\langle E_{k, x}^{1}\right\rangle / U_{p}$ and $\left\langle E_{k, x}^{2}\right\rangle / U_{p}$ are $35 \%$ and $52 \%$ for an intensity of $3.8 \times 10^{15} \mathrm{~W} \mathrm{~cm}^{-2}$ and are smaller than the respective ratios for the smaller intensities. The velocities of electrons 1 and 2 along the direction of the electric field are determined mostly by the values of the vector potential at the tunneling and recollision times, respectively. As a result, the two electrons can escape opposite each other along the direction of the electric field. This pattern is indeed seen in the plots of the correlated momenta in Fig. 4(c5) with high density in the second and fourth quadrants. This antiparallel pattern was predicted in the context of strongly driven $\mathrm{N}_{2}$ with fixed nuclei [31]. It was also seen in the case of Ar driven by intense ultrashort laser fields [24], in agreement with experiment [25].

For single ionization of $\mathrm{He}$ and $\mathrm{Xe}$, it was shown that using the tunneling times as the starting point, the 3D-SMND with all Coulomb forces switched off yields the correct order of magnitude for $\left\langle p_{y}^{1}\right\rangle_{\mathrm{SI}}$. For double ionization of He and $\mathrm{Xe}$, using the 3D-SMND model with all Coulomb forces switched off and with initial conditions taken to be the recollision times and velocities, $\left\langle p_{y}^{1}\right\rangle_{\mathrm{DI}}$ and $\left\langle p_{y}^{2}\right\rangle_{\mathrm{DI}}$ are obtained and presented in Table II. These values of $\left\langle p_{y}^{1}\right\rangle_{\mathrm{DI}}$ and $\left\langle p_{y}^{2}\right\rangle_{\mathrm{DI}}$ agree very well with the values obtained using the $3 \mathrm{D}-\mathrm{SMND}$ model with all Coulomb forces accounted for (see Table II). This agreement further supports that recollision is the main factor determining $\left\langle p_{y}^{1}+p_{y}^{2}\right\rangle_{\mathrm{D} !}$.

Finally, in what follows, the electron that contributes the most to the maximum value of $\left\langle p_{y}^{1}+p_{y}^{2}\right\rangle_{D I}$ is identified for both driven $\mathrm{He}$ and $\mathrm{Xe} .\left\langle p_{y}^{1}\right\rangle_{\mathrm{DI}}$ and $\left\langle p_{y}^{2}\right\rangle_{\mathrm{DI}}$ are plotted as a function of time in Fig. 4, with time zero set equal to the recollision time of each double-ionization trajectory. It is shown in Figs. 4(a4), 4(b4), and 4(c4) that it is mainly $\left\langle p_{y}^{1}\right\rangle_{\mathrm{DI}}$ that changes significantly at the recollision time. This change is sharper for the smaller intensities [Figs. 4(a4) and 4(b4)]. In Fig. 4(b4), at intensity $2.0 \times 10^{15} \mathrm{~W} \mathrm{~cm}^{-2}$, it is also illustrated that in the absence of the magnetic field both $\left\langle p_{y}^{1}\right\rangle_{\text {DI }}$ and $\left\langle p_{y}^{2}\right\rangle_{\mathrm{DI}}$ tend to zero with time, as expected. In addition, in Figs. 3(c) and 3(d), for driven He and Xe, respectively, $\left\langle p_{y}^{1}\right\rangle_{\text {DI }}$ and $\left\langle p_{y}^{2}\right\rangle_{\mathrm{DI}}$ are plotted as a function of intensity. It is seen that $\left\langle p_{y}^{1}\right\rangle_{\text {DI }}$ and $\left\langle p_{y}^{1}+p_{y}^{2}\right\rangle_{D I}$ have maxima around the same intensities. At these intensities $\left\langle p_{y}^{1}\right\rangle_{\text {DI }}$ is significantly larger 
TABLE II. Double-ionization results for $\mathrm{Xe}$ and $\mathrm{He}$.

\begin{tabular}{|c|c|c|c|c|c|c|c|c|c|}
\hline \multirow{2}{*}{\multicolumn{2}{|c|}{$I\left(10^{15} \mathrm{~W} \mathrm{~cm}^{-2}\right)$}} & \multicolumn{4}{|c|}{ NSDI $c_{1,2,3}=1, Z=2$} & \multicolumn{4}{|c|}{$\begin{array}{c}\text { NSDI } c_{1,2,3}=0, \\
t_{0}=t_{\mathrm{rec}}, \mathbf{p}_{0}^{1}=\mathbf{p}_{\mathrm{rec}}^{1}, \mathbf{p}_{0}^{2}=\mathbf{p}_{\mathrm{rec}}^{2}\end{array}$} \\
\hline & & $\left\langle p_{y}^{2}\right\rangle^{\mathrm{a}}$ & $\left\langle E_{k}^{2} / c\right\rangle^{\mathrm{a}}$ & $\left\langle p_{y}^{1}\right\rangle^{\mathrm{a}}$ & $\left\langle E_{k}^{1} / c\right\rangle^{\mathrm{a}}$ & $\left\langle p_{y}^{2}\right\rangle^{\mathrm{a}}$ & $\left\langle E_{k}^{2} / c\right\rangle^{\mathrm{a}}$ & $\left\langle p_{y}^{1}\right\rangle^{\mathrm{a}}$ & $\left\langle E_{k}^{1} / c\right\rangle^{\mathrm{a}}$ \\
\hline \multirow[t]{3}{*}{$\mathrm{He}$} & 0.7 & 12 & 14 & 25 & 16 & 13 & 16 & 25 & 17 \\
\hline & 1.3 & 22 & 22 & 80 & 21 & 24 & 25 & 82 & 22 \\
\hline & 4.8 & 25 & 32 & 73 & 47 & 33 & 39 & 74 & 48 \\
\hline \multirow[t]{3}{*}{$\mathrm{Xe}$} & 0.05 & 10 & 11 & 65 & 9 & 11 & 12 & 67 & 10 \\
\hline & 0.07 & 14 & 13 & 80 & 10 & 15 & 15 & 81 & 10 \\
\hline & 0.22 & 38 & 27 & 30 & 17 & 45 & 35 & 30 & 18 \\
\hline
\end{tabular}

a Average momentum and kinetic energy are given in $10^{-3}$ a.u.

than $\left\langle p_{y}^{2}\right\rangle_{\mathrm{DI}}$. Moreover, it is shown in Figs. 3(c) and 3(d) that once $\left\langle p_{y}^{1}\right\rangle_{\mathrm{DI}}$ reaches a maximum at $2 \times 10^{15} \mathrm{~W} \mathrm{~cm}^{-2}$ for $\mathrm{He}$ and at $10^{14} \mathrm{~W} \mathrm{~cm}^{-2}$ for $\mathrm{Xe}$, it then decreases with increasing intensity for the intensities considered. Thus, $\left\langle p_{y}^{1}\right\rangle_{\mathrm{DI}}$, the average momentum of the tunneling electron, is the one affected the most by strong recollisions.

\section{CONCLUSIONS}

It was shown that the average sum of the electron momenta along the propagation direction of the laser field has large values at intensities where strong recollisions underlie double ionization. This is an unexpected result. For He driven by a near-infrared laser field and for Xe driven by a mid-infrared laser field, the intensities where the average sum of the electron momenta along the propagation direction of the laser field is maximum are smaller than the intensities where magnetic-field effects are predicted to be large. Thus, recollision probes magnetic-field effects at smaller intensities than expected. However, it can also be stated that a magnetic field probes strong recollisions through the measurement of the sum of the electron momenta along the propagation direction of the laser field. It is expected that the findings reported in this work will serve as motivation for future studies. Such studies can identify, for instance, the effect the magnetic field has on the different mechanisms of nonsequential double ionization, i.e., on direct and delayed double ionization and the wavelengths where the magnetic field has the largest effect on recollisions.

\section{ACKNOWLEDGMENTS}

A.E. is grateful for fruitful discussions with P. Corkum. She also acknowledges EPSRC Grant No. J0171831 and the use of the computational resources of Legion at UCL.
[1] K. T. Taylor, J. S. Parker, D. Dundas, and K. J. Meharg, J. Mod. Opt. 54, 1959 (2007).

[2] A. Becker, R. Dörner, and R. Moshammer, J. Phys. B 38, S753 (2006).

[3] D. B. Milošević, G. G. Paulus, D. Bauer, and W. Becker, J. Phys. B 39, R203 (2006).

[4] H. R. Reiss, Phys. Rev. Lett. 101, 043002 (2008).

[5] H. R. Reiss, J. Phys. B 47, 204006 (2014).

[6] S. Palaniyappan, A. DiChiara, E. Chowdhury, A. Falkowski, G. Ongadi, E. L. Huskins, and B. C. Walker, Phys. Rev. Lett. 94, 243003 (2005).

[7] C. H. Keitel and P. L. Knight, Phys. Rev. A 51, 1420 (1995).

[8] C. C. Chirilă, N. J. Kylstra, R. M. Potvliege, and C. J. Joachain, Phys. Rev. A 66, 063411 (2002).

[9] M. W. Walser, C. H. Keitel, A. Scrinzi, and T. Brabec, Phys. Rev. Lett. 85, 5082 (2000).

[10] M. Yu. Emelin and M. Yu. Ryabikin, Phys. Rev. A 89, 013418 (2014).

[11] S. Chelkowski, A. D. Bandrauk, and P. B. Corkum, Phys. Rev. Lett. 113, 263005 (2014).

[12] S. Chelkowski, A. D. Bandrauk, and P. B. Corkum, Phys. Rev. A 92, 051401(R) (2015).

[13] A. S. Titi and G. W. F. Drake, Phys. Rev. A 85, 041404(R) (2012).
[14] I. A. Ivanov, Phys. Rev. A 91, 043410 (2015).

[15] C. T. L. Smeenk, L. Arissian, B. Zhou, A. Mysyrowicz, D. M. Villeneuve, A. Staudte, and P. B. Corkum, Phys. Rev. Lett. 106, 193002 (2011)

[16] P. B. Corkum, Phys. Rev. Lett. 71, 1994 (1993).

[17] B. Wolter, M. G. Pullen, M. Baudisch, M. Sclafani, M. Hemmer, A. Senftleben, C. D. Schröter, J. Ullrich, R. Moshammer, and J. Biegert, Phys. Rev. X 5, 021034 (2015).

[18] A. Ludwig, J. Maurer, B. W. Mayer, C. R. Phillips, L. Gallmann, and U. Keller, Phys. Rev. Lett. 113, 243001 (2014).

[19] A. Emmanouilidou, Phys. Rev. A 78, 023411 (2008).

[20] J. S. Parker, B. J. S. Doherty, K. T. Taylor, K. D. Schultz, C. I. Blaga, and L. F. Di Mauro, Phys. Rev. Lett. 96, 133001 (2006).

[21] A. Staudte, C. Ruiz, M. Schöffler, S. Schössler, D. Zeidler, Th. Weber, M. Meckel, D. M. Villeneuve, P. B. Corkum, A. Becker, and R. Dörner, Phys. Rev. Lett. 99, 263002 (2007).

[22] A. Rudenko, V. L. B. de Jesus, Th. Ergler, K. Zrost, B. Feuerstein, C. D. Schroter, R. Moshammer, and J. Ullrich, Phys. Rev. Lett. 99, 263003 (2007).

[23] A. Emmanouilidou, J. S. Parker, L. R. Moore, and K. Taylor, New J. Phys. 13, 043001 (2011).

[24] A. Chen, M. Kübel, B. Bergues, M. F. Kling, and A. Emmanouilidou, arXiv:1702.04232. 
[25] M. Kübel, C. Burger, N. G. Kling, T. Pischke, L. Beaufore, I. Ben-Itzhak, G. G. Paulus, J. Ullrich, T. Pfeifer, R. Moshammer, M. F. Kling, and B. Bergues, Phys. Rev. A 93, 053422 (2016).

[26] P. Kustaanheimo and E. Stiefel, J. Reine Angew. Math. 218, 204 (1965).

[27] L. D. Landau and E. M. Lifshitz, Quantum Mechanics (Pergamon, New York, 1977).

[28] N. B. Delone and V. P. Krainov, J. Opt. Soc. Am. B 8, 1207 (1991).

[29] E. Yakaboylu, M. Klaiber, H. Bauke, K. Z. Hatsagortsyan, and C. H. Keitel, Phys. Rev. A 88, 063421 (2013).
[30] R. Abrines and I. C. Percival, Proc. Phys. Soc. London 88, 861 (1966).

[31] A. Emmanouilidou and A. Staudte, Phys. Rev. A 80, 053415 (2009).

[32] B. Feuerstein, R. Moshammer, D. Fischer, A. Dorn, C. D. Schröter, J. Deipenwisch, J. R. Crespo Lopez-Urrutia, C. Höhr, P. Neumayer, J. Ullrich, H. Rottke, C. Trump, M. Wittmann, G. Korn, and W. Sandner, Phys. Rev. Lett. 87, 043003 (2001).

[33] R. Kopold, W. Becker, H. Rottke, and W. Sandner, Phys. Rev. Lett. 85, 3781 (2000). 